\title{
APLICAÇÃO DE FITORREGULADORES QUÍMICOS NA QUALIDADE TECNOLÓGICA DO SORGO SACARINO CV. BIOMATRIX 535
}

\author{
RONALDO SILVA VIANA ${ }^{1}$, PAULO ALEXANDRE MONTEIRO FIGUEIREDO ${ }^{1}$, \\ LUCAS APARECIDO MANZANI LISBOA ${ }^{1}$, ANA CAROLINA NUNES DOMINGUES \\ ASSUMPÇÃO ${ }^{1}$, MARCO EUSTÁQUIO SÁ ${ }^{1}$ e ANDRÉ MAY ${ }^{1}$
}

${ }^{1}$ Unesp - campus dracena, ronaldodsv@hotmail.com, paulofigueiredo@dracena.unesp.br, lisboa@dracena.unesp.br,anacndomingues@terra.com.br,marcosa@agr.feis.unesp.br,andre.may@embrapa.br

Revista Brasileira de Milho e Sorgo, v.14, n.3, p. 326-334, 2015

\begin{abstract}
RESUMO - Este trabalho buscou avaliar a aplicação dos fitorreguladores químicos na qualidade tecnológica do sorgo sacarino. O ensaio foi realizado na Usina Cerradinho, localizada em Catanduva-SP. O delineamento experimental utilizado foi em blocos casualizados, no esquema de parcelas subdivididas, em que os tratamentos foram constituídos pela combinação de dez tratamentos envolvendo as aplicações dos fitorreguladores químicos: 5; 10; $20 \mathrm{~g} \mathrm{ha}^{-1}$ sulfome- $^{-}$ turon-methyl; 0,$10 ; 0,20 ; 0,40 \mathrm{~L} \mathrm{ha}^{-1}$ glifosate; 0,$2 ; 0,4 ; 0,8 \mathrm{~L} \mathrm{ha}^{-1}$ ethyl-trinexapac e testemunha. Nos subtratamentos, foram realizadas as amostragens para as avaliações da qualidade tecnológica em épocas distintas após aplicação dos maturadores químicos. A área foi dividida em quatro repetições, cada parcela foi formada de cinco linhas espaçadas de 0,75 m entre si, por $10 \mathrm{~m}$ de comprimento, constituída pela cultivar de sorgo sacarino Biomatrix 535. Foram avaliadas qualidades tecnológicas como: pureza (\% caldo); açúcares redutores; açúcares redutores totais; brix (\% caldo); pol (\% caldo); açúcares teóricos recuperáveis; e fibra (\%). Os fitorreguladores químicos glifosate na dose de $0,40 \mathrm{~L} \mathrm{ha}^{-1}$ e ethyltrinexapac nas dosagens de 0,20; 0,40 e 0,80 $\mathrm{L} \mathrm{ha}^{-1}$ promoveram maior incremento na qualidade tecnológica do sorgo sacarino. As épocas de colheita não influenciaram nas características tecnológicas do sorgo sacarino.
\end{abstract}

Palavras-chave: hormônios, maturadores químicos, etanol.

\section{APPLICATION OF CHEMICAL GROWTH REGULATORS ON THE TECHNOLOGICAL QUALITY OF SWEET SORGHUM CV. BIOMATRIX 535}

\begin{abstract}
This study aimed to evaluate the effect of the application of chemical plant growth regulators on technological quality of sweet sorghum. The assay was performed in the Cerradinho mill located in Catanduva-SP, using the cultivar 535 Biomatrix of sweet sorghum , The experimental design was a randomized block in a split plot, with 10 treatments involving the application of chemical growth regulators: $5 ; 10 ; 20 \mathrm{~g} \mathrm{ha}^{-1}$ sulfometuron-methyl; 0.10 ; $0.20 ; 0.40 \mathrm{~L} \mathrm{ha}^{-1}$ glyphosate; $0.2 ; 0.4 ; 0.8 \mathrm{~L} \mathrm{ha}^{-1}$ ethyl-trinexapac and control. Samplings for assessment of quality were performed at different times after application of the chemical maturators. The area was divided into four blocks (replications), each plot consisted of five $10 \mathrm{~m}$ long rows spaced $0.75 \mathrm{~m}$. Broth purity, reducing sugars, total reducing sugar, Brix (\% juice), Pol (broth), theoretical recoverable sugar and fiber (\%) were evaluated. Glyphosate at $0.40 \mathrm{~L}$ $\mathrm{ha}^{-1}$ and ethyl-trinexapac at $0.20 ; 0.40$ and $0.80 \mathrm{~L} \mathrm{ha}^{-1}$ caused the highest increases on technological quality of sweet sorghum. Harvest time did not affect the technological characteristics of sweet sorghum.
\end{abstract}

Key words: hormones,chemical maturators, ethanol. 
No Brasil, a matéria-prima utilizada com maior rendimento por área cultivada para a produção de etanol, açúcar e rapadura é a cana-de-açúcar. Todavia, continuam as buscas por culturas que sirvam como matérias-primas renováveis e limpas, com a finalidade de minimizar a emissão dos gases do efeito estufa por meio da fixação de carbono, e que apresentem ciclos produtivos curtos. Dentre essas culturas, uma que se destaca é o sorgo sacarino (Borges et al. 2010).

O sorgo Sorghum bicolor L. Moench é uma planta que pode ser uma opção no plantio em período de entressafra da cultura da cana-de-açúcar. As características que mais se destacam no sorgo sacarino são o fato de ser uma cultura de verão de ciclo curto e a tolerância à limitação hídrica. Por ser tratar de uma planta de característica rústica, o sorgo pode ser semeado em áreas onde a cana-de-açúcar não se adaptaria bem (Ceres, 2010). No entanto, para desenvolver todo seu potencial genético de exploração de água e nutrientes, são necessárias condições do solo favoráveis ao desenvolvimento do sistema radicular, principalmente tolerância à acidez do solo, por se tratar de uma espécie relativamente sensível à toxicidade de alumínio (Costa, 2011).

O sorgo sacarino pode servir como uma estratégia para as grandes empresas do setor sucroenergético, podendo ser cultivado durante a reforma do canavial ou mesmo no período da entressafra, apresentando um crescimento rápido com seu ciclo de cultivo de 120 dias, reduzindo o período de entressafra da usina, no qual há considerável ociosidade nas destilarias (Durães, 2011).

Cabe ressaltar que o sorgo sacarino necessita de menor quantidade de fertilizantes no plantio, armazena açúcar em seus colmos em diferentes épocas do ano e apresenta custo de produção do açúcar semelhante ao da cana-de-açúcar (Ceres, 2010).
Definidos como reguladores vegetais, os fitorreguladores alteram a morfologia e a fisiologia da planta, podendo levar a modificações qualitativas e quantitativas na produção agrícola. Além disso, promovem a diminuição do crescimento da planta, possibilitando incrementos do teor de sacarose, precocidade de maturação, aumento na produtividade. Também atuam sobre as enzimas invertases que catalisam o acúmulo de sacarose nos colmos (Caputo et al., 2007).

Vários produtos vêm sendo empregados na cultura nos últimos anos, sendo os principais: glifosate; etil-trinexapac; e sulfometuron methyl. Leite et al. (2009), estudando a qualidade tecnológica, a produtividade e a margem de contribuição agrícola da cana-de-açúcar em função da aplicação de reguladores vegetais no início da safra, concluíram que os fitorreguladores, de forma geral, propiciaram melhoria na qualidade tecnológica da matéria-prima, com reflexo positivo na produtividade de açúcar e na margem de contribuição agrícola.

Diante do exposto, o objetivo deste trabalho foi avaliar a aplicação de fitorreguladores químicos na qualidade tecnológica do sorgo sacarino.

\section{Materiais e métodos}

O ensaio foi instalado e conduzido na Usina Cerradinho S/A, no município de Catanduva-SP, em área de reforma do canavial localizada na Latitude $21^{\circ}$ 5' 59,673", Longitude 48 58'46,961" e $454 \mathrm{~m}$ acima do nível do mar. A área experimental apresentava uma topografia semiplana e o solo da área é classificado como Latossolo Vermelho Férrico Eutrófico. Por ocasião do plantio, foi realizada a correção do solo com calcário dolomítico com PRNT 90\% para elevação da saturação por bases a $60 \%$, segundo Raij 
et al. (1996). As recomendações foram baseadas nas análises de solo descritas na Tabela 1.

O plantio do sorgo sacarino, cultivar Biomatrix 535 , ocorreu entre meados de dezembro de 2011 e janeiro de 2012. O delineamento experimental utilizado foi em blocos casualizados em parcelas subdivididas com quatro repetições, constituído pela combinação de dez tratamentos, envolvendo fitorreguladores em nove situações e uma testemunha, segundo demons- trado na Tabela 2. Como subtratamentos, foram consideradas as coletas de amostras de sorgo sacarino em diferentes épocas. Cada parcela continha cinco linhas com 10 m de comprimento cada. Após 60 dias da semeadura, foram realizadas as aplicações dos fitorreguladores químicos, seguindo o período de aplicação recomendado pelos fabricantes. Foi utilizado um pulverizador com $\mathrm{CO}_{2}$ pressurizado, com uma barra de $6 \mathrm{~m}$ de comprimento, em forma de T, com seis bicos

TABELA 1. Análise química do solo nas profundidades de 0 - 20 e 20 - $40 \mathrm{~cm}$ antes da implantação do experimento. Catanduva-SP, 2011

\begin{tabular}{|c|c|c|}
\hline & & \\
\hline & $0-20$ & $20-40$ \\
\hline $\mathrm{pH} \mathrm{CaCl}{ }_{2}$ & 5,6 & 5,2 \\
\hline $\mathrm{MO} \mathrm{g} \mathrm{dm}{ }^{-3}$ & 13 & 9 \\
\hline $\mathrm{P} \mathrm{mg} \mathrm{dm}^{-3}$ (resina) & 2 & 3 \\
\hline $\mathrm{K} \mathrm{mmol}_{\mathrm{c}} \mathrm{dm}^{-3}$ (resina) & 2,7 & 2,5 \\
\hline Ca mmol $\mathrm{dm}^{-3}$ (resina) & 13 & 12 \\
\hline $\mathrm{Mg} \mathrm{mmol}_{\mathrm{c}} \mathrm{dm}^{-3}$ (resina) & 7 & 7 \\
\hline $\mathrm{H}+\mathrm{Al} \mathrm{mmol} \mathrm{dm}^{-3}$ & 18 & 20 \\
\hline Al mmol $\mathrm{dm}^{-3}$ & 0 & 0 \\
\hline Soma de bases $\mathrm{mmol}_{\mathrm{c}} \mathrm{dm}^{-3}$ & 23 & 22 \\
\hline CTC mmol $\mathrm{dm}^{-3}$ & 41 & 42 \\
\hline Saturação por bases \% & 56 & 52 \\
\hline Saturação Al \% & 0 & 0 \\
\hline $\mathrm{S}\left(\mathrm{SO}^{-2}\right) \mathrm{mg} \mathrm{dm}^{-3}$ & 8 & 11 \\
\hline $\mathrm{Cu} \mathrm{mg} \mathrm{dm}{ }^{-3}$ (DTPA) & 0,6 & 0,8 \\
\hline Fe mg dm (DTPA) & 15 & 11 \\
\hline $\mathrm{Zn} \mathrm{mg} \mathrm{dm}{ }^{-3}$ (DTPA) & 0,4 & 0,4 \\
\hline Mn mg dm ${ }^{-3}$ (DTPA) & 14,0 & 8,8 \\
\hline B mg dm-3 (água quente) & 0,15 & 0,28 \\
\hline Argila $\mathrm{g} \mathrm{kg}^{-1}$ & 113 & 137 \\
\hline Silte $\mathrm{g} \mathrm{kg}^{-1}$ & 37 & 33 \\
\hline Areia Total $\mathrm{g} \mathrm{kg}^{-1}$ & 850 & 830 \\
\hline Areia Grossa & 150 & 120 \\
\hline Areia Fina & 700 & 710 \\
\hline
\end{tabular}

MO: matéria orgânica; CTC: capacidade de troca de cátions. 
AXI 11002 com jato do tipo plano, possibilitando a aplicação simultânea em duas linhas. A barra foi colocada horizontalmente apoiada sobre outras duas barras verticais que mantinham a barra pulverizadora a, aproximadamente, $50 \mathrm{~cm}$ acima do dossel. A pressão utilizada foi constante de 40 libras $\mathrm{Pol}^{2}$ com volume de $200 \mathrm{~L} \mathrm{ha}^{-1}$, segundo Viana et al. (2008). As aplicações foram realizadas durante o período de 07h00min às 11 h00min. Na ocasião, havia pouca ocorrência de ventos e a temperatura ambiente encontrava-se ao redor de 25 a $30^{\circ} \mathrm{C}$, com umidade relativa do ar entre 60 e $80 \%$.

Em quatro épocas, ou seja, aos 15, 22, 37 e 44 dias após aplicação do fitorregulador (DAA), foram realizadas avaliações previstas para os subtratamentos. Em cada época, foram coletadas manualmente dez plantas sequenciais em linha de plantio de sorgo sacarino. As plantas foram despontadas na altura da gema apical, ou seja, ponto de quebra, e enviadas ao Laboratório de Pagamento da Usina Cerradinho, no município de Catanduva-SP para realização das análises tecnológicas. O processamento foi realizado segundo a metodologia do Sistema de Pagamento pelo
Teor de Sacarose (PCTS). Após a desintegração e a homogeneização dos colmos, uma alíquota de $500 \mathrm{~g}$ foi submetida à prensa hidráulica. $\mathrm{O}$ caldo extraído foi destinado para o laboratório para a determinação de: Brix (\% caldo); Pol (\% caldo); Açúcar Teórico Recuperável (ATR); Fibra (\%); Pureza (\% caldo); Açúcares Redutores - AR (\%); e Açúcares Redutores Totais - ART (\%), utilizando os métodos de determinações químico-tecnológicas, conforme Circular Consecana (2006).

As variáveis foram submetidas à análise de variância pelo teste $\mathrm{F}(\mathrm{p}<0,05)$ e comparadas pelo teste de Tukey a $5 \%$ de probabilidade, sendo utilizado o programa Assistat 7.6 Beta (Silva; Azevedo, 2002).

\section{Resultados e Discussão}

Os resultados obtidos com a cultivar de sorgo sacarino Biomatrix 535 para as características tecnológicas Brix (\% caldo), Pol (\% caldo), Açúcar Teórico Recuperável (ATR) e Fibra (\%) nos tratamentos com fitorreguladores, nas diferentes épocas de amostragens, estão contidos na Tabela 3.

TABELA 2. Fitorreguladores químicos aplicado aos 65 dias após o plantio, cultivar de sorgo sacarino Biomatrix 535. Catanduva-SP, 2013.

\begin{tabular}{clc}
\hline Tratamento & \multicolumn{1}{c}{ Ingrediente Ativo } & Dose (L ou g p.c. ha $\left.{ }^{-1}\right)$ \\
\hline 1 & sulfometuron-methyl & 5 \\
2 & sulfometuron-methyl & 10 \\
4 & sulfometuron-methyl & 20 \\
5 & glifosate & 0,10 \\
6 & glifosate & 0,20 \\
7 & glifosate & 0,40 \\
8 & ethyl-trinexapac & 0,20 \\
9 & ethyl-trinexapac & 0,40 \\
10 & ethyl-trinexapac & 0,80 \\
\hline
\end{tabular}


TABELA 3. Valores médios observados para Brix (\% caldo), Pol (\% caldo), Açúcar Teórico Recuperável (ATR) e Fibra (\%) nos tratamentos com fitorreguladores nas diferentes épocas de amostragens na cultivar de sorgo sacarino Biomatrix 535. Catanduva-SP, 2013.

\begin{tabular}{lllll}
\hline Causas de variação & Brix (\% caldo) & Pol (\% caldo) & ATR & Fibra (\%) \\
\hline Fitorreguladores (A) & & & & \\
sulfometuron methyl $5 \mathrm{~g} \mathrm{ha}^{-1}$ & $12,04 \mathrm{~d}$ & $3,40 \mathrm{e}$ & $48,41 \mathrm{e}$ & $12,69 \mathrm{e}$ \\
sulfometuron methyl $10 \mathrm{~g} \mathrm{ha}^{-1}$ & $12,02 \mathrm{~d}$ & $3,12 \mathrm{e}$ & $46,56 \mathrm{e}$ & $12,45 \mathrm{e}$ \\
sulfometuron methyl $20 \mathrm{~g} \mathrm{ha}^{-1}$ & $14,05 \mathrm{c}$ & $4,67 \mathrm{de}$ & $59,51 \mathrm{de}$ & $13,32 \mathrm{e}$ \\
glifosate 0,10 L ha- & $16,36 \mathrm{~b}$ & $5,56 \mathrm{~cd}$ & $66,64 \mathrm{~cd}$ & $14,16 \mathrm{~cd}$ \\
glifosate 0,20 L ha-1 & $15,08 \mathrm{bc}$ & $4,48 \mathrm{de}$ & $58,00 \mathrm{de}$ & $13,30 \mathrm{de}$ \\
glifosate 0,40 L ha-1 & $19,95 \mathrm{a}$ & $8,35 \mathrm{a}$ & $90,56 \mathrm{a}$ & $16,01 \mathrm{a}$ \\
ethyl-trinexapac 0,20 L ha-1 & $16,74 \mathrm{~b}$ & $6,97 \mathrm{abc}$ & $77,70 \mathrm{abc}$ & $15,26 \mathrm{abc}$ \\
ethyl-trinexapac 0,40 L ha & $16,27 \mathrm{~b}$ & $7,41 \mathrm{ab}$ & $81,39 \mathrm{ab}$ & $14,97 \mathrm{abc}$ \\
ethyl-trinexapac 0,80 L ha-1 & $16,97 \mathrm{~b}$ & $7,69 \mathrm{ab}$ & $83,28 \mathrm{a}$ & $15,74 \mathrm{ab}$ \\
Testemunha & $13,56 \mathrm{~cd}$ & $6,10 \mathrm{bcd}$ & $68,93 \mathrm{bcd}$ & $14,64 \mathrm{bcd}$ \\
DMS & 1,97 & 1,70 & 14,31 & 1,36 \\
\hline Épocas (B) & & & \\
(15daa) & $15,50 \mathrm{a}$ & $5,91 \mathrm{a}$ & $69,27 \mathrm{a}$ & $14,31 \mathrm{a}$ \\
(22 daa) & $14,88 \mathrm{a}$ & $5,68 \mathrm{a}$ & $67,42 \mathrm{a}$ & $13,95 \mathrm{a}$ \\
(37 daa) & $15,82 \mathrm{a}$ & $5,95 \mathrm{a}$ & $69,58 \mathrm{a}$ & $14,55 \mathrm{a}$ \\
(44 daa) & $15,02 \mathrm{a}$ & $5,55 \mathrm{a}$ & $66,12 \mathrm{a}$ & $14,21 \mathrm{a}$ \\
DMS & 1,02 & 0,67 & 5,64 & 0,61 \\
Fator A & $36,38 * *$ & $26,80 * *$ & $25,73 * *$ & $20,12 * *$ \\
Fator B & $2,45 \mathrm{~ns}$ & $1,12 \mathrm{~ns}$ & $1,14 \mathrm{~ns}$ & $2,21 \mathrm{~ns}$ \\
Fator A x B & $6,30 * *$ & $5,76 * *$ & $5,88 * *$ & $3,24 * *$ \\
\hline CV Parcela & 10,67 & 24,49 & 17,40 & 7,92 \\
CV sub Parcela & 11,47 & 19,90 & 14,15 & 7,37 \\
\hline
\end{tabular}

Letras iguais não diferem entre si nas colunas pelo Teste de Tukey dentro do mesmo fator $(\mathrm{P}=0,05)$; ${ }^{\mathrm{P}}<0,05 ; * * \mathrm{P}<0,01 ;$ ns $\mathrm{P} \geq$ 0,05 ; (daa) dias após aplicação.

O tratamento com regulador vegetal glifosato e na dosagem de $0,40 \mathrm{~L} \mathrm{ha}^{-1}$ favoreceu um incremento nos valores médios das características tecnológicas Brix, Pol e Fibra, independentemente das épocas de amostragens realizadas. Para essas características, os outros fitorreguladores, com suas respectivas dosagens, apresentaram resultados inferiores.
Para indução artificial da maturação, o glifosato paralisa o crescimento da altura da planta e modifica a partição dos fotoassimilados, deslocando-os para o acúmulo de sacarose. O modo de ação deste fitorregulador ocorre através da utilização de dosagens reduzidas que inibe a via metabólica do ácido chiquímico, de acordo com Meschede et al. (2011). 
De acordo com Prasad et al. (2007), o estágio ideal de colheita da cana-de-açúcar ocorre quando o caldo apresenta entre 15,5 e $16,5^{\circ}$ Brix, sendo este grau importante para obtenção de um caldo com alta qualidade de fermentação, consequentemente com maximização na produção de etanol por hectare. Outros autores aconselham médias mais altas de Brix para colheita (Almodares; Hadi, 2009). Segundo Kumar et al. (2008), o Brix pode ser influenciado pela adubação ou pela fertilidade do solo ou até mesmo pelo uso de fitorreguladores químicos.

Silva et. al. (2010) relataram que o fitorregulador sulfometuron methyl, quando aplicado em diferentes variedades de cana-de-açúcar, possibilitou melhoria da qualidade tecnológica, ou seja, determinou resposta significativa com relação a ganhos de Pol, aumentos da Pureza e redução no teor de ácidos orgânicos do caldo. Os resultados são concordantes com Leite et al. (2009) para os valores de Pol.

Os teores de fibra encontrados neste trabalho seguiram os resultados relatados por Silva et al. (2010), que também apontam aumento dessa característica na cana-de-açúcar por meio da aplicação dos fitorreguladores glifosato e sulfometuron methyl.

Para a característica ATR, os fitorreguladores glifosato $0,4 \mathrm{~L} \mathrm{ha}^{-1}$ e ethyl-trinexapac $0,80 \mathrm{~L} \mathrm{ha}^{-1}$ proporcionaram as maiores médias. Os valores são parcialmente semelhantes aos relatados por Silva et al. (2010) que, em estudo sobre fitorreguladores químicos em cana-de-açúcar aplicados em final de safra, constataram um aumento significativo sobre a característica tecnológica ATR ao longo das épocas de colheitas, quando utilizados glifosato $0,4 \mathrm{~L} \mathrm{ha}^{-1} \mathrm{e}$ sulfometuron methyl $20 \mathrm{~g} \mathrm{ha}^{-1}$.

$\mathrm{O}$ valor pago pela matéria-prima aos produtores está diretamente expresso no ATR encontrado. Sua qualidade é afetada pelo ambiente e pelas condi- ções de manejo da cultura. Neste sentido, é observada diretamente a influência dos fitorreguladores sob os valores de ATR na cultura após a colheita (Meschede et al. 2009; Souza \& Foloni, 2012).

Os resultados obtidos com a cultivar de sorgo sacarino Biomatrix 535 para as características tecnológicas Pureza (\% caldo), Açúcares Redutores - AR (\%) e Açúcares Redutores Totais - ART (\%) nos tratamentos com fitorreguladores, nas diferentes épocas de amostragens, estão contidos na Tabela 4.

Para a característica Pureza, os melhores resultados encontrados foram os tratamentos testemunha e ethyl-trinexapac $0,40 \mathrm{~L} \mathrm{ha}^{-1}$ e ethyl-trinexapac $0,80 \mathrm{~L} \mathrm{ha}^{-1}$, que apresentaram diferença significativa entre si. $\mathrm{O}$ fator época não influenciou os resultados encontrados para essa característica. Podemos dizer que, quanto maior a pureza da cana, melhor a qualidade da matéria-prima para se recuperar açúcar. Estes resultados corroboram com Fernandes et al. (2002), que também determinaram maiores valores de pureza após a aplicação de sulfometuron metil.

Para a característica Açúcares Redutores, o melhor resultado encontrado foi o tratamento com sulfometuron methyl $10 \mathrm{~g} \mathrm{ha}^{-1}$. Para Açúcares Redutores Totais, os melhores resultados foram com glifosato $0,40 \mathrm{~L} \mathrm{ha}^{-1}$ e com ethyl-trinexapac $0,80 \mathrm{~L} \mathrm{ha}^{-1}$. Para essas características avaliadas, o fator época não influenciou os resultados encontrados. As concentrações de açúcares redutores totais (ART) presentes na cultivar testada são similares aos valores encontrados para as cultivares $27 \mathrm{~B}$ x kellar e $27 \mathrm{~B} \mathrm{x}$ BJ 248 (Audilakshmi et al., 2010). Por outro lado, os valores são superiores aos encontrados por Leite et al. (2009) quando estudaram cana-de-açúcar, o que corrobora com Ribeiro Filho et al. (2008). Meschede et al. (2011) relatam que o sulfumeturon-methyl com doses elevadas pode interferir na fotossíntese devido 
TABELA 4. Valores médios observados para Pureza (\% caldo), Açúcares Redutores - AR (\%) e Açúcares Redutores Totais - ART (\%) nos tratamentos com fitorreguladores nas diferentes épocas de amostragens na cultivar de sorgo sacarino Biomatrix 535. Catanduva-SP, 2013.

\begin{tabular}{|c|c|c|c|c|}
\hline Causas de variação & Pureza ( $\%$ caldo $)$ & AR $(\%)$ & ART $(\%)$ & Fibra $(\%)$ \\
\hline \multicolumn{5}{|l|}{ Fitorreguladores (A) } \\
\hline sulfometuron methyl $5 \mathrm{~g} \mathrm{ha}^{-1}$ & $32,31 \mathrm{c}$ & $2,11 \mathrm{ab}$ & $5,69 \mathrm{e}$ & $12,69 \mathrm{e}$ \\
\hline sulfometuron methyl $10 \mathrm{~g} \mathrm{ha}^{-1}$ & $29,70 \mathrm{c}$ & $2,20 \mathrm{a}$ & $5,49 \mathrm{e}$ & $12,45 \mathrm{e}$ \\
\hline sulfometuron methyl $20 \mathrm{~g} \mathrm{ha}^{-1}$ & $35,96 \mathrm{c}$ & $1,99 \mathrm{ab}$ & $6,91 \mathrm{de}$ & $13,32 \mathrm{e}$ \\
\hline glifosato $0,10 \mathrm{~L} \mathrm{ha}^{-1}$ & $39,77 \mathrm{bc}$ & $1,85 b$ & $7,70 \mathrm{~cd}$ & $14,16 \mathrm{~cd}$ \\
\hline glifosato $0,20 \mathrm{~L} \mathrm{ha}^{-1}$ & $34,14 \mathrm{c}$ & $2,04 \mathrm{ab}$ & $6,76 \mathrm{de}$ & $13,30 \mathrm{de}$ \\
\hline glifosato $0,40 \mathrm{~L} \mathrm{ha}^{-1}$ & $49,49 \mathrm{ab}$ & $1,53 \mathrm{c}$ & $10,32 \mathrm{a}$ & $16,01 \mathrm{a}$ \\
\hline ethyl-trinexapac $0,20 \mathrm{~L} \mathrm{ha}^{-1}$ & $49,38 \mathrm{ab}$ & $1,55 \mathrm{c}$ & $8,89 \mathrm{abc}$ & $15,26 \mathrm{abc}$ \\
\hline ethyl-trinexapac $0,40 \mathrm{~L} \mathrm{ha}^{-1}$ & $52,94 \mathrm{a}$ & $1,47 \mathrm{c}$ & $9,27 \mathrm{ab}$ & $14,97 \mathrm{abc}$ \\
\hline ethyl-trinexapac $0,80 \mathrm{~L} \mathrm{ha}^{-1}$ & $55,14 \mathrm{a}$ & $1,38 \mathrm{c}$ & $9,49 \mathrm{a}$ & $15,74 \mathrm{ab}$ \\
\hline Testemunha & $52,91 \mathrm{a}$ & $1,47 \mathrm{c}$ & $7,89 \mathrm{bcd}$ & $14,64 \mathrm{bcd}$ \\
\hline DMS & 10,16 & 0,29 & 1,53 & 1,36 \\
\hline \multicolumn{5}{|l|}{ Épocas (B) } \\
\hline (15daa) & $43,54 a$ & $1,75 \mathrm{a}$ & $7,97 \mathrm{a}$ & $14,31 \mathrm{a}$ \\
\hline (22 daa) & $43,16 a$ & $1,77 \mathrm{a}$ & $7,76 \mathrm{a}$ & $13,95 \mathrm{a}$ \\
\hline (37 daa) & $43,76 a$ & $1,73 \mathrm{a}$ & $8,01 \mathrm{a}$ & $14,55 \mathrm{a}$ \\
\hline (44 daa) & $42,23 a$ & $1,79 \mathrm{a}$ & $7,63 \mathrm{a}$ & $14,21 \mathrm{a}$ \\
\hline DMS & 4,21 & 0,12 & 0,61 & 0,61 \\
\hline Fator A & $21,49 * *$ & $26,31 * *$ & $26,55^{* *}$ & $20,12 * *$ \\
\hline Fator B & $0,35 \mathrm{~ns}$ & $0,48 \mathrm{~ns}$ & $1,18 \mathrm{~ns}$ & $2,21 \mathrm{~ns}$ \\
\hline Fator A x B & $3,24 * *$ & $3,57 * *$ & $5,97 * *$ & $3,24 * *$ \\
\hline CV Parcela & 19,49 & 13,69 & 16,24 & 7,92 \\
\hline CV sub Parcela & 16,67 & 12,03 & 13,31 & 7,37 \\
\hline
\end{tabular}

Letras iguais não diferem entre si nas colunas pelo Teste de Tukey dentro do mesmo fator $(\mathrm{P}=0,05) ;{ }^{*} \mathrm{P}<0,05 ; * * \mathrm{P}<0,01 ; \mathrm{nsP} \geq 0,05$; (daa) dias após aplicação.

a diminuição dos teores de clorofila e carotenóides nas folhas. Os autores ressaltam que análises de clorofila e, principalmente, de carotenóides podem ser um bom indicador para determinar os efeitos de fitotoxicidade desses produtos no vegetal.

Os valores de ART obtidos neste trabalho são semelhantes a aqueles encontrados por Ribeiro Filho et al. (2008), que realizaram a moagem dos colmos do sorgo sacarino com as folhas. De acordo com a literatura, a redução de sólidos solúveis presentes no caldo após 34 dias de florescimento está relacionada com a conversão destes compostos, principalmente de açúcares em amido presentes nos grãos, que servirão como material de reserva ou convertidos em material fibroso sob a forma de celulose (Tsuchihashi; Goto, 2004). 
Vale destacar que, na cultura da cana-de-açúcar, Silva e Segato (2011) enfatizam que o emprego de fitorreguladores químicos pode ser uma importante ferramenta na obtenção de melhores características tecnológicas devido a ação dos produtos que são aplicados com a finalidade de antecipar o processo de maturação. Além disso, promovem melhorias na qualidade da matéria-prima a ser processada, aperfeiçoam os resultados agroindustriais e econômicos, além de auxiliar no planejamento da safra, permitindo maior eficiência no manejo da cultura.

\section{Conclusão}

De maneira geral, a aplicação de fitorreguladores na cultura do sorgo sacarino proporcionou aumento das características tecnológicas. As épocas de colheita não influenciaram nas características tecnológicas do sorgo sacarino.

\section{Referências}

AUDILAKSHMI,S.;MALL,A.K.;SWARNALATHA, M.;http://www.sciencedirect.com/science/article/ pii/S0961953410000395 SEETHARAMA, N. Inheritance of sugar concentration in stalk (Brix), sucrose content, stalk and juice yield in sorghum.

Biomass and Bioenergy, Oxford, v. 34, p. 813820, 2010.

ALMODARES, A.; HADI, M. R. Production of bioethanol from sweet sorghum: a review.

African Journal of Agricultural Research, Washington, v. 4, n. 9, p. 772-780, 2009.

BORGES, I. D.; MENDES, A. A.; VIANA, E. J.; GUSMÃO, C. A. G.; RODRIGUES, H. F. F.; CARLOS, L. de A. Caracterização do caldo extraído dos colmos da cultivar de sorgo sacarino BRS 506 (Sorghum bicolor L.). In: CONGRESSO NACIONAL DE MILHO E SORGO, 28.; SIMPÓSIO BRASILEIRO SOBRE A LAGARTA DO CARTUCHO, 4., 2010, Goiânia. Potencialidades, desafios e sustentabilidade: resumos expandidos... Goiânia: ABMS, 2010. 1 CD-ROM. CERES SEMENTES DO BRASIL. Sorgo sacarino tem vantagens que o diferenciam da cana de açúcar. Centralina, 2010. Disponível em: $<$ http://www.ceres.net/ceressementes/Etanol/ EtanolVantagens.html >. Acesso em 12 ago. 2015. CAPUTO, M. M.; SILVA, M. de A. S.; BEAUCLAIR, E. G. F.; GAVA, G. J. de C.; Acúmulo de sacarose, produtividade e florescimento de cana-de-açúcar sob reguladores vegetais. Interciência, Caracas, v. 32, n. 12, p. 834-840, 2007.

CONSELHO DOS PRODUTORES DE CANA-DEAÇÚCAR, AÇÚCAR E ÁLCOOL DO ESTADO DE SÃO PAULO. Manual de instruções. 5. ed. Piracicaba, 2006. 200 p.

COSTA, C. H. M. da. Efeito residual da aplicação superficial de calcário e gesso nas culturas de soja, aveia-preta e sorgo granífero. $2011.80 \mathrm{f}$. Dissertação (Mestrado) - Faculdade de Ciências Agronômicas, Universidade Estadual Paulista, Botucatu, 2011.

DURÃES, F. O. M. Sorgo sacarino: desenvolvimento de tecnologia agronômica. Agroenergia em Revista, Brasília, ano 2, n. 3, p. 7, ago. 2011.

FERNANDES, A. C.; STUPIELLO, J. P.; UCHOA, P. E. A. Utilização do Curavial para melhoria da qualidade da cana-de-açúcar.STAB: açúcar, álcool esubprodutos, Piracicaba, v.20,n.4,p.43-45,2002. FORNASIERI FILHO, D; FORNASIERI, J. L. Manual da cultura do sorgo. Jaboticabal: Funep, 2009. 202 p. 
LEITE, G. H. P.; CRUSCIOL, C. A. C.; SILVA, M. de A.; VENTURINI FILHO, W. G. Technological quality of sugarcane uppon application of ripeners during the cropping season. Bragantia, Campinas, v. 68, n. 2, p. 527-534, 2009.

MESCHEDE, D. K.; VELINI, E. D.; CARBONARI, C. A.; SILVA, J. R. M. Physiological alteration in sugarcane influenced by glyphosate and sulfumeturon-methyl application. Planta Daninha, Viçosa, MG, v. 29, n. 2,p.413-419, 2011. MESCHEDE, D. K.; CARBONARI, C. A.; VELINI, E. D. Different chemical ripeners action on sugarcane yield and technological quality. Revista Brasileira de Herbicidas, Brasília, v. 8, n. 2, p. 62-67, 2009.

KUMAR, S. R.; SHROTRIA, P. K.; DESHMUKH, J. P. Characterizing nutrient management effect on yield of sweet sorghum genotypes. World Journal of Agricultural Sciences, Pradesh, v. 4, n. 6, p. 787-789, 2008.

PRASAD, S.; ANOOP S.; JAIN, N.; JOSHI, H. C. Ethanol production from sweet sorghum syrup for utilization as automotive fuel in India. Energy and Fuels, Washington, v. 21, p. 24152420, 2007.

RAIJ, B. van; CANTARELLA, H. Outras culturas industriais. In: RAIJ, B. van; CANTARELLA, H.; QUAGGIO J. A.; FURLANI, A. M. C. (Coord.). Recomendações de adubação e calagem para o Estado de São Paulo. 2. ed. Campinas: Instituto Agronômico, 1996. p. 233-236. RIBEIRO FILHO, N. M.; ALVES, R. M.; FLORÊNCIO, I. M.; FLORENTINO, E. R.; DANTAS, J. P. Viabilidade de utilização do caldo do sorgo sacarino para a produção de álcool carburante (etanol). Disponível em: $<$ http://annq.org/congresso2007/trabalhos_
apresentados/T59.pdf>. Acesso em: 12 ago. 2015.

SILVA, F. de A. S.; AZEVEDO, C. A. V. Versão do programa computacional Assistat para o sistema operacional Windows. Revista Brasileira de Produtos Agroindustriais, Campina Grande, v. 4, n. 1, p. 71-78, 2002.

SILVA, M. de A.; GAVA, G. J. C.; CAPUTO, M. M.; SORRILLA, P. F.; BASSETTO, S. C. Productivity of stalk and sucrose in sugarcane in relation to cultivar and ripener interaction. Scientia Agraria, Curitiba, v. 11, n. 4, p. 323329, 2010.

SILVA, R. F. S.; SEGATO, S. V. Importância do uso de maturadores vegetais na cultura da cana-deaçúcar. Nucleus, Ituverava, v. 8, n. 2, p. 35-46, 2011.

SOUZA, R. A.; FOLONI, L. L. Uso de biostimulantes como maturadores de cana-de-açúcar. In: CONGRESSO BRASILEIRO DA CIÊNCIA DAS PLANTAS DANINHAS, 28., 2012, Campo Grande. A ciência das plantas daninhas na era da biotecnologia: anais. Campo Grande: SBCPD, 2012. p. 338-342.

TSUCHIHASHI, N.; GOTO, Y. Cultivation of sweet sorghum (Sorghum bicolor (L.) Moench) and determination of its harvest time to make use as the raw material for fermentation, practiced during rainy season in dry land of Indonesia. Plant Production Science, n. 7, p. 442-448, 2004.

VIANA, R. da S.; SILVA, P. H.; MUTTON, M. A.; MUTTON, M. J. R.; GUIMARÃES, E.

R.; BENTO, M. Efeito da aplicação de maturadores químicos na cultura da cana de açúcar (Saccharum spp.) variedade SP 81-3250. Acta Science Agronomy, Maringá, v. 30, n. 1, p. 6571, 2008. 